\title{
Perfiles motivacionales de elección de estudios en estudiantes universitarios de nuevo ingreso*
}

Career Choice Motivational Profiles in First Year University Students

\author{
MARÍA InÉs GARCÍA-RIPA ${ }^{a}$ \\ Pontificia Universidad Católica Argentina, Argentina \\ ORCID: http://orcid.org/0000-0001-8582-7573 \\ María Fe SÁnchez-García \\ Universidad Nacional de Educación a Distancia, \\ España \\ Angélica RisQuez \\ Univiversidad de Limerick, Irlanda
}

\footnotetext{
a Autor de correspondencia. Correo electrónico: ines_ripa@uca.edu.ar
}

Para citar este artículo: García-Ripa, M., I., Sánchez-García, M. F., \& Risquez, A. (2018). Perfiles motivacionales de elección de estudios en estudiantes universitarios de nuevo ingreso. Universitas Psychologica, 17(3), 1-12. https://doi.org/ 10.11144/Javeriana.upsy17-3.pmee

\section{RESUMEN}

Este trabajo tuvo como propósito analizar los motivos de elección de estudios y establecer perfiles motivacionales diferenciados en estudiantes universitarios de nuevo ingreso en la Universidad Católica Argentina (UCA). Para ello se diseñó y validó un cuestionario ad hoc, estructurado en tres escalas de motivación: (1) Motivos de superación profesional y altruismo; (2) Búsqueda de prestigio y solvencia económica y (3) Motivos de evitación. El cuestionario se aplicó a la totalidad de los estudiantes de nuevo acceso a la UCA en el curso académico 2013-2014 (1 156 estudiantes de diferentes disciplinas). Los resultados permitieron establecer la existencia de grupos diferenciados de estudiantes y obtener un modelo predictivo de clasificación destinado al diseño de acciones de orientación que tengan en cuenta sus motivaciones y necesidades en un contexto determinado.

\section{Palabras clave}

motivación; elección de estudios; género; edad; área disciplinar; universidad.

\footnotetext{
ABSTRACT

This study examines the career choice motivations of first year students in the Catholic University of Argentina (UCA) and describes a classification of the student characteristics that are associated with these motivations. A questionnaire was designed and validated for this purpose. The factor analysis renders three motivational dimensions: (1) Self-fulfilment and altruism; (2) Search for prestige and economic welfare; and (3) Avoidance motivation. The instrument has been applied to the first year cohort at UCA in the academic year 2013/14 (a total of 1156 students from different disciplines). The existence of differentiated student groups resulting from scores in the motivational scales has been demonstrated. The resulting predictive model can be purposed to guide interventions that are mindful of students' motivations and needs in a particular context.

Keywords

motivation; career choice; gender; age; discipline of study; university.
} 
La decisión de iniciar estudios universitarios implica un proceso de búsqueda y selección donde los estudiantes priorizan ciertos motivos de elección de estudios que pueden responder tanto a valoraciones y motivaciones propias como a factores externos reflejados, por ejemplo, en expectativas familiares o estereotipos sociales (Abarca, Gormaz, \& Leiva, 2012; Navarro Guzmán \& Casero Martínez, 2012; Valle Arias et al., 2010). Las razones que manifiestan los estudiantes al momento de elegir una carrera universitaria pueden estar fundamentadas en actitudes motivacionales hacia el aprendizaje y hacia la vida profesional de carácter intrínseco, o bien tener un carácter más extrínseco (González Maura, 2004; Grouzet et al., 2005; Ryan \& Deci, 2000). Por ejemplo, la elección de unos estudios por la búsqueda de recompensas externas o la proyección de ciertos resultados, tales como alcanzar el reconocimiento y prestigio profesional $\mathrm{u}$ obtener un empleo bien remunerado, son entendidos como motivos extrínsecos (Gámez, Marrero, \& Díaz, 2008). En cambio, los motivos más intrínsecos estarían asociados a actividades que en sí mismas tienen una recompensa o que responden a la búsqueda de la realización personal, como el deseo de aprender o el logro de un desarrollo personal y profesional a través de los estudios universitarios (Cano Celestino, 2008). Los estudiantes, en el momento de elegir una opción de estudio superior, pueden sostener en forma simultánea una serie de razones y combinar motivos intrínsecos y extrínsecos en diversos grados (González, Paoloni, Donolo, \& Rinaudo, 2012). Esto a la vez puede incidir en el posterior desarrollo de la carrera profesional, tanto en cuanto al desempeño como a la continuidad de los estudios y, posteriormente, en las habilidades de adaptación al mundo laboral (Ye, 2015). Se ha comprobado que aquellos jóvenes que presentan una motivación más intrínseca obtienen un mejor rendimiento académico y se sienten orgullosos de los logros obtenidos (González et al., 2012; Mancini, Caricati, Panari, \& Tonarelli, 2015; Valle Arias et al., 2010), alcanzan un mayor proceso de autodeterminación profesional (González Maura, 2004), tienen un mayor compromiso y eficiencia (García Garduño, 2007) y muestran menos intenciones de abandonar sus estudios (Mancini et al., 2015). Aquellos, en cambio, que lo hacen por motivos extrínsecos como razones económicas, tradición familiar u otros- tienden a mostrar menor compromiso con su desempeño y menor confianza en lo que hacen (Avendaño Bravo \& González Urrutia, 2012; Ryan \& Deci, 2000).

Algunos estudios muestran que los motivos de elección de estudios aparecen asociados con el género y la disciplina escogida (Gámez et al., 2008; Navarro Guzmán \& Casero Martínez, 2012). Por ejemplo, se han hallado evidencias de que los motivos como la búsqueda de afiliación con otras personas, los intereses personales o de altruismo, la búsqueda de poder, el sentimiento de logro o el sostenimiento económico difieren según se trate de estudiantes mujeres o varones (Abarca et al., 2012; Gámez \& Marrero, 2003; Navarro Guzmán \& Casero Martínez, 2012). A su vez, los estudiantes de ciertas disciplinas privilegian la búsqueda de prestigio en la elección de su formación universitaria en relación con otros motivos (González López, 2005).

Todo lo expuesto hace pensar en la importancia de conocer, respecto a los estudiantes que ingresan a la universidad, los motivos que orientan su elección hacia una $u$ otra profesión y la conveniencia de establecer mecanismos de orientación para fortalecer aquellos motivos que regulan mejor la actuación del estudiante en su trayectoria formativa (González Maura, 2004). Algunos autores como Skatova y Ferguson (2014) advierten que la literatura actual no ha explorado los motivos de elección de carrera suficientemente. Además, las diferencias encontradas en los estudios en función del sexo, edad y área disciplinar no son lo bastante claras y consistentes. Es importante, igualmente, que los hallazgos recogidos en la literatura no sean extrapolados automática e incuestionablemente a contextos específicos, sin tener en cuenta variables culturales, socioeconómicas e institucionales propias.

En este estudio, se propone un análisis de los motivos de elección de carrera de estudiantes argentinos en la Universidad Católica Argentina 
(UCA) que puede resultar de utilidad en contextos culturales cercanos. Este trabajo atiende a los siguientes objetivos: (1) Analizar los motivos que los estudiantes de nuevo ingreso identifican como más significativos en relación con la elección de sus estudios universitarios; (2) Determinar si entre estos estudiantes existen perfiles motivacionales según el género, la edad o el área disciplinar y (3) Establecer un modelo predictivo que permita clasificar a los estudiantes en los grupos o perfiles identificados.

Ya que la hipótesis de partida establece la existencia de diferencias motivacionales a la hora de iniciar los estudios, lo que tendría implicaciones para la orientación en ese momento, la identificación de las características diferenciales vinculadas a las motivaciones de elección permitirá entonces plantear intervenciones de apoyo y asesoramiento contextualmente relevantes con el fin de incidir, desde un enfoque preventivo, en el óptimo desarrollo de su formación universitaria.

\section{Método y materiales}

El estudio adopta un enfoque descriptivo, exploratorio e inferencial, buscando identificar diferentes perfiles motivacionales de elección de estudios, en función del sexo, el rango de edad y el área disciplinar de los estudiantes. Con el fin de disponer de un instrumento adaptado al contexto institucional y a los objetivos del estudio, se diseñó el Cuestionario de Motivos de Elección de Carrera (CMEC), para el que se tomaron en cuenta aportes de algunos cuestionarios relativos a esta temática (Gámez \& Marrero, 2003; Migone de Faletty \& Moreno, 1999), así como los resultados de otros estudios más recientes (Abarca et al., 2012; Avendaño Bravo \& González Urrutia, 2012; Valle Arias et al., 2010). Para comprobar las características psicométricas del cuestionario, previamente se realizó un estudio de su validez de constructo (mediante análisis factorial) y de su fiabilidad (alpha de Cronbach), obteniéndose resultados satisfactorios, según se recoge en el apartado de resultados.
El cuestionario final consta de 20 ítems iniciales relativos a los motivos de elección de estudios, construidos en una escala tipo Likert sobre el grado de prioridad que se adjudica a cada uno de los motivos. Considerando la revisión teórica efectuada, los ítems se estructuraron en torno a 2 tipos de motivación: intrínseca (16 ítems) y extrínseca (6 ítems).

El cuestionario se aplicó a una muestra de 1156 estudiantes de nuevo ingreso de la Pontificia Universidad Católica Argentina (UCA), pertenecientes a distintas áreas disciplinares (Tabla 1). Dicha muestra se corresponde con la población total de los estudiantes que accedieron a la UCA en el curso académico 2013-2014, los cuales fueron invitados a cumplimentar el cuestionario en el contexto de las sesiones de tutoría, organizadas por el Departamento de Ingreso de la universidad.

\section{Tabla 1}

Distribución de la muestra de estudiantes según sexo, edad y área disciplinar

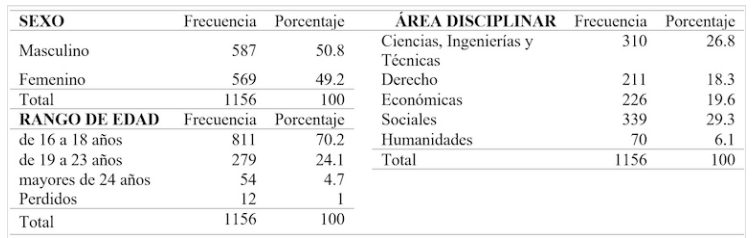

La comprobación de la hipótesis establecida se llevó a cabo mediante un estudio multivariado de análisis de conglomerados y discriminativo no jerárquico con la finalidad de identificar grupos o perfiles de motivación. Como técnica multivariante, el análisis de conglomerados o clústeres permite formar grupos de elementos o variables sobre la base de una máxima homogeneidad y diferenciación entre los mismos (García Pérez, 2005). En este caso, se ha elegido el método no jerárquico que asegura la mejor clasificación de los individuos de la muestra, y se puede considerar un procedimiento adecuado para establecer perfiles en muestras numerosas de estudiantes (Valle Arias et al., 2010). Este método, a diferencia del análisis jerárquico, implica que se establezca a priori la cantidad de grupos que se desea obtener. 
Una vez clasificados los perfiles de estudiantes respecto a cada uno de los conglomerados obtenidos, se buscó establecer si el sexo, el área disciplinar o el rango de edad explicaban el tipo de conglomerado obtenido, para lo cual se realizó un análisis de dependencia a través del estudio discriminante, comparando los resultados observados en cada caso, a un nivel de significación $\alpha \leq 0.01$. Los análisis estadísticos se efectuaron con la ayuda del software SPSS versión 24.

\section{Resultados}

\section{Estudio de la validez y fiabilidad del cuestionario}

Respecto a las características psicométricas del instrumento se llevó a cabo en primer lugar el estudio de su estructura factorial, a través del método de Ejes Principales con rotación Varimax que permite identificar el tipo de varianza de los ítems del cuestionario. Las medidas previas de $\mathrm{KMO}(0.818)$ y prueba de Bartlett (0) confirman la idoneidad del análisis factorial.

Sobre los 26 ítems iniciales del cuestionario se eliminaron aquellos que en la rotación de las comunalidades obtuvieron un índice menor al inicial. Dado que los resultados indican la conformación de seis factores, donde algunos de ellos solo tienen uno o dos ítems, se procedió a realizar un nuevo análisis factorial, observándose mejores valores en los ítems rotados (Tabla 2).
Tabla 2

Resultados del análisis factorial para la validez de constructo

\begin{tabular}{|c|c|c|c|}
\hline \multirow[b]{2}{*}{ Descripción Ítems } & \multicolumn{3}{|c|}{ Factor } \\
\hline & 1 & 2 & 3 \\
\hline Crees que estudiar esta carrera te ayudará a comunicarte mejor con los demás. & & & \\
\hline Quieres conocer más sobre los temas particulares que trata la carrera. & 0.67 & & \\
\hline Crees que el esfuerzo que realices para estudiar será productivo para la sociedad. & 0.64 & & \\
\hline Es una forma de superar un reto importante en tu vida. & 0.5 & & \\
\hline Comprendes el valor social que tiene esta carrera. & 0.54 & & \\
\hline Quieres hacer algo que te haga feliz y satisfecho contigo mismo. & 0.68 & & \\
\hline Aunque se te presenten varios obstáculos, es lo que quieres estudiar. & 0.68 & & \\
\hline Valoras tus capacidades y quieres mejorar cada dia. & 0.71 & & \\
\hline $\begin{array}{l}\text { Siempre te han llamado la atención las profesiones donde se pueda supervisar y } \\
\text { organizar actividades. }\end{array}$ & & 0.54 & \\
\hline Querrias tener un puesto de trabajo con cierto prestigio social. & & 0.74 & \\
\hline $\begin{array}{l}\text { Una vez recibido, tendrás mucha oferta laboral y podrás tener un buen pasar } \\
\text { económico. }\end{array}$ & & 0.71 & \\
\hline Te permitirá acceder a trabajos bien remunerados. & & 0.65 & \\
\hline Quieres ocupar un cargo directivo. & & 0.68 & \\
\hline $\begin{array}{l}\text { Puede ser una manera de acceder a una posición dominante en tu desarrollo } \\
\text { profesional. }\end{array}$ & & 0.6 & \\
\hline Te evitará sentirte fracasado/a en la vida. & & & 0.64 \\
\hline No puedes quedarte en tu casa sin hacer nat & & & 0. \\
\hline Crees que te va a resultar fácil cursarla. & & & 0. \\
\hline No querias quedarte sin estudiar algo. & & & 0.64 \\
\hline No es muy dificil de estudiar. & & & 0.50 \\
\hline $\begin{array}{l}\text { Te consideras una persona con problemas y a través de la carrera dejarás de } \\
\text { tenerlos. }\end{array}$ & & & 0.51 \\
\hline
\end{tabular}

Te consideras una persona con problemas y a través de la carrera dejarás de

Notas.Método de extracción: factorización del eje principal. Método de rotación: normalización Varimax con Kaiser. La rotación ha convergido en cuatro iteraciones.

El análisis factorial permite identificar la existencia de tres factores que justifican el $42.75 \%$ de la varianza acumulada total. El primer factor (que explica el $21.61 \%$ de la varianza) se ha denominado Motivos de superación profesional y altruismo y remite a motivos intrínsecos de elección de estudios al logro de situaciones agradables; el segundo factor (13.47 \% de la varianza) Búsqueda de prestigio y solvencia económica, incluye motivos relativos al logro de un reconocimiento externo tanto desde la retribución monetaria como del lugar de poder y valoración externa, por lo que tendría un carácter más extrínseco. El tercer factor $(7.66 \%$ de la varianza), denominado Motivos de evitación, agrupa los ítems basados en la evitación de situaciones indeseables, cuya naturaleza intrínseca o extrínseca resulta más ambigua. El análisis de fiabilidad a través del índice alpha de Cronbach aporta un valor global de 0.82 , así como de 0.792 para el primer factor, de 0.801 para el segundo y de 0.797 para el tercero, valores que permiten considerar como aceptable la fiabilidad del instrumento. 
Diferencias motivacionales en la elección de estudios

Seguidamente, el análisis de conglomerados, a través del método no jerárquico, permitió identificar los motivos de elección de estudios que prevalecen en los estudiantes de nuevo ingreso, configurándose cinco grupos diferentes. Para la interpretación del nivel de prevalencia de cada motivo se identificó la media obtenida en cada uno de los tres motivos estudiados y se estableció la siguiente clasificación: los valores medios obtenidos en cada uno de los motivos, entre 1 y 3 se interpretaron como una baja valoración por ese motivo; las medias obtenidas entre 3 y 4 , se definieron como valoración media y los valores entre 4 y 6 puntos se establecieron como valoración alta. De esta forma, se comparan los cinco conglomerados obtenidos de acuerdo a la valoración (alta, media o baja) de cada uno de los motivos estudiados (Tabla 3).

Tabla 3

Resultados de clasificación de conglomerados

\begin{tabular}{|c|c|c|c|c|c|}
\hline \multirow[b]{2}{*}{ Motivos } & \multicolumn{5}{|c|}{ Conglomerados } \\
\hline & $\begin{array}{c}1 \\
(\mathrm{n}=238)\end{array}$ & $\begin{array}{c}2 \\
(\mathrm{n}=201)\end{array}$ & $\begin{array}{c}3 \\
(\mathrm{n}=212)\end{array}$ & $\begin{array}{c}4 \\
(n=267)\end{array}$ & $\begin{array}{c}5 \\
(n=225)\end{array}$ \\
\hline $\begin{array}{l}\text { 1-Motivos de superación } \\
\text { personal y altruismo }\end{array}$ & $\begin{array}{r}3.84 \\
\text { (media) }\end{array}$ & $\begin{array}{r}4.96 \\
\text { (alta) }\end{array}$ & $\begin{array}{r}4.98 \\
\text { (alta) }\end{array}$ & $\begin{array}{r}5.16 \\
\text { (alta) }\end{array}$ & $\begin{array}{r}4.33 \\
\text { (alta) }\end{array}$ \\
\hline $\begin{array}{l}\text { 2-Búsqueda de prestigio y } \\
\text { solvencia económica }\end{array}$ & $\begin{array}{r}3.98 \\
\text { (media) }\end{array}$ & $\begin{array}{r}3.21 \\
\text { (media) }\end{array}$ & $\begin{array}{r}4.37 \\
\text { (alta) }\end{array}$ & $\begin{array}{r}4.79 \\
\text { (alta) }\end{array}$ & $\begin{array}{r}2.66 \\
\text { (baja) }\end{array}$ \\
\hline 3-Motivos de evitación & $\begin{array}{r}3.02 \\
\text { (media) }\end{array}$ & $\begin{array}{r}3.31 \\
\text { (media) }\end{array}$ & $\begin{array}{r}2.16 \\
\text { (baja) }\end{array}$ & $\begin{array}{r}3.90 \\
\text { (media) }\end{array}$ & $\begin{array}{r}1.80 \\
\text { (baja) }\end{array}$ \\
\hline
\end{tabular}

La comparación de medias, mediante el método de pares simples, de cada uno los motivos de elección de estudios (en cada conglomerado), permite apreciar una variación muy significativa entre los motivos, en los conglomerados 3, 4 y 5 . Solo en el conglomerado 1 existe una diferencia significativa entre los motivos de superación personal y búsqueda de prestigio $(t=-3.325, p$ $\leq 0.01$ ) y en el conglomerado 2 no se observa una diferencia significativa entre los motivos de búsqueda de prestigio y los motivos de evitación.

Como segundo estudio de análisis, se realizó un contraste de los conglomerados, a través de la comparación de medias, respecto a sexo, disciplina y rango de edad. Respecto a la distribución por sexo, se observa que existe una diferencia en la cantidad de estudiantes varones o mujeres, en cada uno de los cinco grupos de conglomerados (Tabla 4). Los motivos de superación personal y altruismo prevalecen en el grupo de estudiantes mujeres ( $M=4.801$, $D E=0.701)$ respecto al de varones $(M=$ $4.511, D E=0.752)$, encontrando una diferencia significativa $(F=44.438, p \leq 0.01)$ entre sus medias, y de manera inversa, el grupo de varones prioriza los motivos de prestigio y solvencia económica $(\mathrm{M}=3.975, \mathrm{DE}=0.905)$ respecto a las mujeres $(\mathrm{M}=3.713, \mathrm{DE}=0.995)$, con una diferencia significativa de sus medias $(F$ $=23.096, p \leq 0.01)$. En cambio, respecto a los motivos de evitación, no se observa una diferencia significativa y es el motivo menos valorado en ambos grupos (Varones: $\mathrm{M}=2.909$, $D E=0.933$; Mujeres: $M=2.84, D E=0.951$ ).

\section{Tabla 4}

Distribución de los estudiantes según sexo

\begin{tabular}{ccrrrrr}
\hline \multirow{2}{*}{ Sexo } & \multirow{2}{*}{ Total casos analizados } & \multicolumn{5}{c}{ Conglomerado } \\
\cline { 3 - 7 } & & 1 & 2 & \multicolumn{1}{c}{3} & \multicolumn{1}{c}{4} & \multicolumn{1}{c}{5} \\
\hline \multirow{2}{*}{ Varones } & 584 & 148 & 62 & 140 & 82 & 152 \\
& Porcentaje & 25.34 & 10.62 & 23.97 & 14.04 & 26.03 \\
\hline \multirow{2}{*}{ Mujeres } & 559 & 105 & 163 & 54 & 131 & 106 \\
& Porcentaje & 18.78 & 29.16 & 9.66 & 23.43 & 18.96 \\
\hline
\end{tabular}

Respecto al área disciplinar, la distribución de los estudiantes de acuerdo a la carrera elegida es muy diferente en cada uno de los conglomerados, lo cual permite discriminar la conformación del tipo de estudiante en cada grupo (Tabla 5). La comparación de medias, según área disciplinar, permite identificar variaciones muy significativas entre los motivos de búsqueda de desarrollo personal y altruismo $(F=20.679, p$ $\leq 0.01)$ y los motivos de búsqueda de prestigio y solvencia económica $(F=46.079, p \leq 0.01)$, pero no así entre los estudiantes de las diversas áreas disciplinares respecto a los motivos de evitación. Existe una mayor valoración por la búsqueda de desarrollo personal y altruismo en los estudiantes de Sociales $(M=4.859 ; \mathrm{DE}=$ 0.696) y Humanidades $(M=4.833 ; D E=0.768)$ respecto de los de Derecho $(M=4.722 ; D E$ $=0.734)$, Ciencias, Ingenierías y Técnicas $(M$ $=4.584 ; \mathrm{DE}=0.676)$ y Económicas $(\mathrm{M}=$ 4.319; $D E=0.766)$. En cambio, los estudiantes de Económicas $(\mathrm{M}=4.352 ; \mathrm{DE}=0.784)$ son los que presentan mayor preferencia por la 
búsqueda de prestigio y solvencia económica al elegir los estudios superiores, respecto de los de Humanidades $(M=2.836 ; D E=0.995)$ y del resto de los estudiantes (Derecho: $\mathrm{M}=3.917$; $D E=0.818$; Ciencias, Ingenierías y Técnicas: $M$ $=3.891 ; \mathrm{DE}=0.928$; Sociales: $\mathrm{M}=3.63 ; \mathrm{DE}$ $=0.945$ ).

\section{Tabla 5}

Distribución de los estudiantes según área disciplinar

\begin{tabular}{|lcccccc|}
\hline \multirow{2}{*}{ Sexo } & \multirow{2}{*}{ Total de casos analizados } & \multicolumn{5}{c|}{ Conglomerado } \\
\cline { 3 - 7 } Ciencias, Ingenierías y Técnicas & & 1 & 3 & 4 & 5 \\
Derecho & 308 & 164 & 0 & 0 & 0 & 144 \\
& Porcentaje & 53.25 & 0 & 0 & 0 & 46.75 \\
Económicas & 210 & 89 & 0 & 7 & 0 & 144 \\
& Porcentaje & 42.38 & 0 & 3.33 & 0 & 54.29 \\
Sociales & 224 & 0 & 14 & 154 & 56 & 0 \\
& Porcentaje & 0 & 6.25 & 68.75 & 25 & 0 \\
Humanidades & 332 & 0 & 160 & 33 & 139 & 0 \\
& Porcentaje & 0 & 48.19 & 9.94 & 41.87 & 0 \\
& 69 & 0 & 51 & 0 & 18 & 0 \\
& Porcentaje & 0 & 73.91 & 0 & 26.09 & 0 \\
\hline
\end{tabular}

Por último, al analizar la comparación de los cinco grupos de conglomerados en función del rango de edad, se observa que en el primer conglomerado hay mayor presencia de estudiantes de entre 19 y 23 años. En el segundo, prevalecen los estudiantes de 24 años en adelante y en el tercer grupo, los de 18 a 22 años (Tabla 6). Los estudiantes mayores a 23 años priorizan los motivos de superación profesional y altruismo $(\mathrm{M}=5.049 ; \mathrm{DE}=0.731)$, observándose, solo en este caso, una diferencia significativa $(F=$ 10.687, $p \leq 0.01$ ) respecto a los otros dos grupos etarios (De 19 a 23 años: $M=4.720$; $D E=0.722$; de 16 a 18 años: $M=4.605$; $D E=0.742)$. En los tres rangos de edad, los estudiantes valoran, en término medio, los motivos de prestigio y solvencia económica (de 19 a 23 años: $M=3.863 ; D E=0.953$; de 16 a 18 años: $M=3.861 ; D E=0.952$ y de 24 años o más: $M=3.543 ; D E=0.731$ ) y de manera muy baja los motivos de evitación (de 19 a 23 años: $M=2.975 ; D E=1.026$; de 16 a 18 años: $M=$ 2.857; $D E=0.9$ y de 24 años o más: $M=2.617$; $D E=1.114)$.
Tabla 6

Distribución de los estudiantes según rango de edad

\begin{tabular}{lcccccc}
\hline \multirow{2}{*}{ Sexo } & \multirow{2}{*}{ Total casos analizados } & \multicolumn{5}{c}{ Conglomerado } \\
\cline { 3 - 7 } & & 1 & 2 & 3 & 4 & 5 \\
\hline De 16 a 18 años & 799 & 182 & 138 & 148 & 180 & 151 \\
& Porcentaje & 22.78 & 17.27 & 18.52 & 22.53 & 19.9 \\
\hline De 19 a 23 años & 278 & 49 & 47 & 52 & 75 & 55 \\
& Porcentaje & 17.63 & 16.91 & 18.71 & 26.98 & 19.78 \\
\hline De 24 años en adelante & 54 & 4 & 12 & 11 & 10 & 17 \\
& Porcentaje & 7.41 & 22.22 & 20.37 & 18.52 & 31.48 \\
\hline
\end{tabular}

Modelo de clasificación mediante análisis discriminante

Como último análisis, se realizó un estudio discriminante por pasos a fin de estimar cuales son las variables que mejor predicen la configuración de los estudiantes en cada uno de los conglomerados hallados, y de esa manera establecer si el modelo puede clasificar de manera fiable casos futuros. Así, se puede identificar no solo qué variables son útiles para la clasificación de los conglomerados, sino también, cuáles de esas variables contribuyen mejor a dicha clasificación. El análisis discriminante se realizó en tres pasos: en el primero, se introdujo la variable motivo de evitación; en el segundo, la búsqueda de prestigio y, por último, el motivo de superación personal, obteniéndose valores estadísticos aceptables para ser introducidos en el modelo de clasificación de los conglomerados (Motivos de evitación: $F=609.217, p \leq 0.01$; Búsqueda de prestigio y solvencia económica: $F$ $=516.935, p \leq 0.01$ y Motivos de superación personal y altruismo: $F=424.126, p \leq 0.01$ ).

De acuerdo con las variables de clasificación, es decir los motivos de elección, se puede visualizar que los mismos permiten una adecuada clasificación y discriminación de los grupos de conglomerados, aunque habría un mayor solapamiento entre el grupo 1 y el 2 (Figura 1). 


\section{Figura 1}

Distribución de los conglomerados a partir de las variables de clasificación.

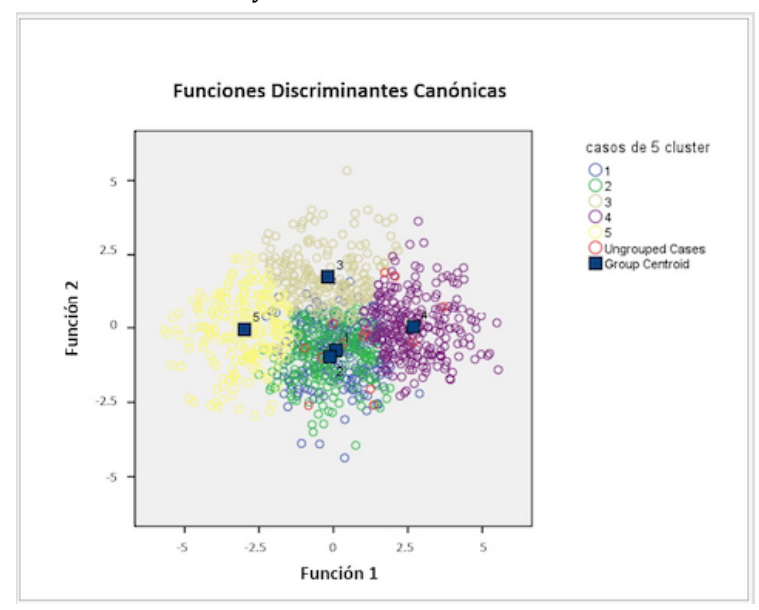

Dado el interés por conocer la probabilidad de que estas mismas variables sirvan como modelo clasificatorio en nuevos estudios, se elaboró la Tabla 7.

Tabla 7

Pronóstico de clasificación de los conglomerados

\begin{tabular}{lrrrrrrr}
\hline \multirow{2}{*}{ Conglomerados } & \multicolumn{9}{c}{ Grupo de pertenencia pronosticado } & Porcentaje de \\
\cline { 2 - 7 } & \multicolumn{1}{r}{1} & 2 & 3 & 4 & 5 & clasificación \\
\hline $1(\mathrm{~N}=238)$ & 232 & 5 & 0 & 1 & 0 & 97.5 \\
$2(\mathrm{~N}=201)$ & 0 & 201 & 0 & 0 & 0 & 100 \\
$3(\mathrm{~N}=212)$ & 0 & 0 & 212 & 0 & 0 & 100 \\
$4(\mathrm{~N}=267)$ & 0 & 1 & 3 & 263 & 0 & 98.5 \\
$5(\mathrm{~N}=225)$ & 5 & 1 & 0 & 0 & 219 & 97.3 \\
\hline Casos no agrupados & 5 & 4 & 1 & 3 & 0 & 20.02 \\
\hline
\end{tabular}

El análisis discriminante permite comprobar que la probabilidad de volver a clasificar los grupos en cada conglomerado es muy alta, dado que el $98.6 \%$ de los casos originales fueron clasificados en el mismo conglomerado inicial. Como síntesis, el análisis de las características que configuran cada conglomerado se puede apreciar a través de la Tabla 8.
Tabla 8

Características predominantes que configuran los conglomerados

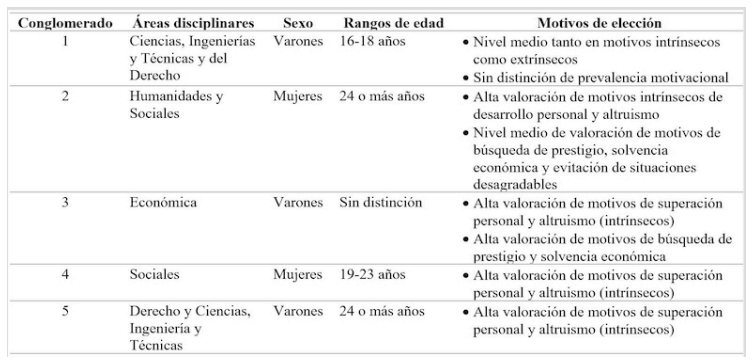

\section{Conclusiones y discusión}

Este trabajo permitió analizar y construir un modelo de clasificación para identificar las diferencias entre los estudiantes que ingresan a la universidad, respecto a los motivos de elección de sus estudios universitarios. A través del análisis de conglomerados se ha podido establecer la configuración de cinco grupos diferenciados de estudiantes en función del grado de prevalencia de cada motivo. Al mismo tiempo, a través de los resultados del estudio discriminante fue posible comprobar que los tipos de motivos de elección de estudios universitarios son adecuados para clasificar a los estudiantes en grupos que son distintivos entre sí y homogéneos internamente.

Un primer grupo de estudiantes (conglomerado 1) está configurado por estudiantes del área de las Ciencias, Ingenierías y Técnicas y del Derecho, en mayor número de varones con edades entre 16 y 18 años, que no presentan una valoración alta en algún motivo de elección de estudios universitarios. Este grupo valora en un nivel medio tanto los motivos intrínsecos de búsqueda de desarrollo personal y motivos de evitación como los motivos extrínsecos de búsqueda de prestigio y solvencia económica. Este hallazgo es comparable con el estudio de Valle Arias et al. (2010) que identifica la existencia de grupos de estudiantes con motivaciones generalizadas sin distinción de la prevalencia de algún motivo sobre los demás. En relación con las variables analizadas en el presente estudio, es razonable asumir que estos estudiantes se encuentran en un proceso de 
elaboración de la propia decisión de estudios universitarios debido a su edad y por ese motivo no consideran ninguna razón de elección por encima de otras opciones.

Un segundo grupo (conglomerado 2) conformado por estudiantes de Humanidades y Sociales, mayoritariamente femenino y con edades mayores a 23 años, prioriza los motivos de desarrollo personal y altruismo, aunque presentan también un nivel medio de preferencia por motivos extrínsecos de búsqueda de prestigio y solvencia económica e intentan elegir sus estudios con el fin de evitar situaciones desagradables a nivel personal. A diferencia del grupo anterior, es probable que la mayor valoración intrínseca sea una distinción propia de estudiantes que eligen áreas disciplinares de carácter más humanístico y que tradicionalmente han tenido una alta representación femenina.

El tercer grupo (conglomerado 3), conformado por estudiantes del área Económica, preferentemente varones y sin gran diferenciación por el rango de edad, valora de manera alta tanto los motivos de superación personal y altruismo como los de búsqueda de prestigio y solvencia económica. Podría pensarse que para este grupo de estudiantes la elección de estudios superiores intenta equilibrar tanto razones intrínsecas de desarrollo personal como extrínsecas de reconocimiento externo y bienestar económico, sin considerarlos motivos de evitación en su elección de estudios universitarios. En forma parecida al estudio de Valle Arias et al. (2010), que identifica la prevalecia de motivos intrínsecos y extrínsecos en uno de sus grupos motivacionales.

El cuarto grupo (conglomerado 4) está conformado por estudiantes de Sociales, preferentemente mujeres de 19 a 23 años que valoran altamente los motivos de superación personal y altruismo. Podría pensarse que, a diferencia del primer grupo, los estudiantes de mayor edad tienen una idea más clara de lo que desean lograr en su desarrollo personal y formación universitaria, a expensas de consideraciones más extrínsecas e instrumentales, aunque esa preferencia puede deberse también a la disciplina de estudio más que a la edad. Lo cual se asemeja más a las investigaciones que plantean diferencias de motivos según disciplinas (González López, 2005), pero no existen comparaciones según rangos de edad.

Por último, el quinto grupo (conglomerado 5) está integrado por estudiantes de Derecho y Ciencias, Ingeniería y Técnicas, mayoritariamente varones de 24 años o más edad, que valoran los motivos de superación personal y altruismo. Comparando esta configuración con la del primer grupo donde coinciden las mismas áreas disciplinares, la diferencia de edad podría ser la explicación de priorizar los motivos intrínsecos que apuntan a un desarrollo personal y no considerar motivos extrínsecos o de evitación en la misma medida. Aunque la variable de edad, se deberá contrastar con otros estudios.

Como característica general se observa que las áreas disciplinares marcan diferencias en la prevalencia de motivos y, en algunos casos, también lo hacen la edad y el sexo. Las áreas de estudio relacionadas con lo humanístico y social son más populares con estudiantes que no valoran tanto los motivos extrínsecos de prestigio y solvencia económica, en contraste con los estudiantes Economía, Ingeniería o Derecho. Esto viene a corroborar hallazgos en otros contextos, donde los motivos intrínsecos prevalecen en carreras sociales y de humanidades (Avendaño Bravo \& González Urrutia, 2012; Gámez et al., 2008; Gámez \& Marrero, 2003) y los extrínsecos en carreras científicas y técnicas (Cano Celestino, 2008; Skatova \& Ferguson, 2014). Es interesante notar que el conjunto de la población estudiantil valora prioritariamente los motivos de superación personal, en consonancia con lo hallado en otros contextos (de Barros, 2015) y que a medida que los estudiantes maduran, la preferencia por estos motivos aumenta considerablemente.

Por último, se aprecia que el sexo mantiene una vinculación con determinadas áreas disciplinares, presentándose, en el caso de la distribución de las mujeres, un abanico más reducido de opciones académicas. En los grupos donde existe un mayor número de estudiantes 
varones, se observa mayor valoración por la búsqueda de prestigio y solvencia económica, en contraste con aquellos donde hay mayor cantidad de estudiantes mujeres, las cuales tienden a valorar preferentemente la superación personal y el altruismo. Estos resultados coinciden con estudios (Abarca et al., 2012; Navarro Guzmán \& Casero Martínez, 2012; Skatova \& Ferguson, 2014) que señalan que la motivación femenina para elegir una carrera hace referencia a la vocación y a factores intrínsecos de interés por el contenido y ayuda a los demás, mientras que en los hombres las carreras son elegidas por motivos más instrumentales tales como el salario, las posibilidades y la proyección profesional.

Los motivos de evitación, que han obtenido puntaciones bajas en la mayoría de las medias de los conglomerados, requieren un análisis particular. Las dos medias más altas se observan en los conglomerados 2 y 4 donde hay una mayor prevalencia de mujeres y de estudiantes de sociales y humanidades. Analizando las razones que configuran este factor, se puede pensar que en estos grupos hay un intento por evitar sentirse fracasados o evitar carreras de mayor esfuerzo, lo cual podría tener un trasfondo de indecisión vocacional e identificarse quizá como un grupo de riesgo de abandono, lo cual requeriría un seguimiento y estudio más pormenorizado de este grupo de estudiantes. Valle Arias et al. (2010) sostienen que los estudiantes con una motivación de evitación del fracaso presentan una preocupación por no parecer incompetentes ante los demás y, en este sentido, los alumnos que eligen estas áreas disciplinares tal vez lo hagan porque interpretan un nivel menor de exigencia respecto de estudios propios de las ciencias e ingenierías. Skatova y Ferguson (2014) también han encontrado que los motivos de elección de carrera de los estudiantes de artes y humanidades venían determinados por interés en la disciplina, pero también por poca preocupación por las salidas profesionales y algún nivel de pereza o desgano (loafing), ya que los estudiantes cursaban estas disciplinas como una alternativa fácil de acceso a la universidad. Por otra parte, la expectativa de lograr solucionar problemas o evitar tenerlos posiblemente también esté muy relacionada con estudios humanísticos o sociales (Rovella, Sans de Uhrlandt, Solares, Delfino, \& Diaz, 2008) que requieren mayor introspección y la construcción de habilidades interpersonales.

Finalmente, es posible que factores socioeconómicos también jueguen un papel en los motivos de evitación. En su reciente estudio, de Barros (2015) comparó los valores vitales de los estudiantes universitarios tras la reciente crisis económica, para juzgar en qué medida son compatibles con actitudes hacia el emprendimiento que podrían facilitar su éxito en un entorno laboral caracterizado por la incertidumbre, la inestabilidad y las transiciones de carrera múltiples. La autora corroboró que, aunque la motivación de logro es un motivador importante, los estudiantes en su muestra son adversos al riesgo y los valores relacionados con el emprendimiento no son especialmente populares. De manera similar, Troiano y Elias (2014) encontraron una relación significativa entre la composición social de los estudios universitarios y las expectativas estudiantiles. Especialmente, las autoras comprobaron que los estudiantes provenientes de clase trabajadora suelen escoger sus estudios por motivos instrumentales y de evitación del riesgo en mayor medida que los estudiantes de extractos sociales más favorecidos, lo que no es sorprendente debido al alto sacrificio económico asociado a la universidad para muchas familias trabajadoras. Estos resultados constituyen un aporte que puede ser considerado en las instancias de orientación frente a los procesos de elección de la carrera universitaria. A diferencia de otros estudios, han demostrado una mayor diferenciación de grupos lo suficientemente heterogéneos entre sí donde las variables sexo, área disciplinar y edad implican diferencias significativas de clasificación.

Se ha logrado construir un modelo de categorización de perfiles estudiantiles de acuerdo con los motivos de elección de estudios superiores con un alto nivel de predictibilidad en futuros estudios semejantes. La posibilidad de contar con este modelo permitirá una mejor identificación de necesidades de orientación tutorial de acuerdo con las motivaciones predominantes. En especial, los resultados 
plantean la necesidad de analizar el proceso de toma de decisiones al inicio de la trayectoria académica universitaria dado que las decisiones académicas, tomadas al finalizar la escuela secundaria, determinan el futuro profesional de cada estudiante (González Afonso, Alvarez Pérez, Cabrera Pérez, \& Bethencourt Benítez, 2007; Navarro Guzmán \& Casero Martínez, 2012). Las implicaciones también atañen al desarrollo de madurez de carrera de los estudiantes durante su estancia en la universidad y lo que está en nuestra mano al respecto, desde el punto de vista docente. Por ejemplo, Kim y Choi (2015) demostraron el impacto que las actividades de instrucción con un elemento creativo pueden tener en la motivación académica y la madurez vocacional, especialmente en autoeficacia, nivel de preferencia de tarea, decisión de carrera y compromiso con la misma.

Considerando las limitaciones del presente estudio, futuras aproximaciones deberían ampliar la muestra de estudiantes a diversas instituciones universitarias en la región y el país, para poder inferir generalizaciones y ofrecer mejores referentes de comparación. Como así también, ampliar el número de variables para un mejor análisis, como considerar variables de personalidad y preferencias personales e historia académica, o el estrato socioeconómico, como se observa en algunos estudios. Al mismo tiempo, sería importante poder explorar en futuros trabajos el tipo de relación establecida entre el perfil motivacional y el desempeño académico posterior en la carrera (Abarca et al., 2012; Cano Celestino, 2008; González et al., 2012), desde un diseño longitudinal.

\section{Referencias}

Abarca, N., Gormaz, N., \& Leiva, P. I. (2012). Expectativas de roles futuros de estudiantes universitarios en Chile. Universitas Psychologica, 11(2), 405-414. Recuperado de http://revistas.javeriana.ed u.co/index.php/revPsycho/article/view/748
Avendaño Bravo, C., \& González Urrutia, R. (2012). Motivos para ingresar a las carreras de Pedagogía de los estudiantes de primer año de la Universidad de Concepción. Estudios Pedagógicos, 2, 21-33. Recuperado de http://mingaonline.uach.cl/pdf/estped/v 38n2/art02.pdf

Cano Celestino, M. A. (2008). Motivación y elección de carrera. Revista Mexicana de Orientación Educativa, 5(13), 6-9. Recuperado de http://remo.ws/revistas/rem o-13.pdf

de Barros, A. F. (2015). The life goals of university-aged students during an ongoing economic and financial crisis. Current Psychology, 34(2), 434-446. https://doi.org/ 10.1007/s12144-014-9267-9

Gámez, E., \& Marrero, H. (2003). Metas y motivos en la elección de la carrera universitaria: un estudio comparativo entre psicología, derecho y biología. Anales de Psicología, 19(1), 121-131. Recuperado de http://www.um.es/analesps/v19/v19_1/1 2-19_1.pdf

Gámez, E., Marrero, H., \& Díaz, J. M. (2008). Motivación interpersonal y metas vitales en estudiantes universitarios. En I. Etxebarría (Ed.), Emoción y motivación: Contribuciones actuales (1.a ed., pp. 173-185). Gipuzcoa, ES: AME.

García Garduño, J. M. E. (2007). Motivación y actitudes hacia la carrera de profesor de educación primaria en estudiantes normalistas de primer ingreso. Revista Mexicana de Orientacion Educativa, 12 (35), 1153-1178. Recuperado de http://www.comie.org.mx/documentos/r mie/v12/n035/pdf/N35C.pdf

García Pérez, A. (2005). Métodos avanzados de estadística aplicada. Técnicas avanzadas. Madrid: Editorial UNED.

González Afonso, M., Álvarez Pérez, P., Cabrera Pérez, L., \& Bethencourt Benítez, J. (2007). El abandono de los estudios universitarios: factores determinantes y medidas preventivas. Revista Española de Pedagogía, 65(236), 71-88. Recuperado 
de https://revistadepedagogia.org/wp-conte nt/uploads/2007/06/236-07.pdf

González López, I. (2005). Motivación y actitudes del alumnado universitario al inicio de la carrera. ¿Varían al egresar? Revista Electrónica de Investigación Psicoeducativa y Psicopedagógica, 3(1), 35-56. Recuperado de http://www.investigacion-psicopedagogi ca.org/revista/new/ContadorArticulo.php? 29

González Maura, V. (2004). La orientación profesional y currículum universitario. Una estrategia educativa para el desarrollo profesional responsable (1. ${ }^{\circ}$ ed.). Barcelona: LAERTES.

González, A., Paoloni, V., Donolo, D., \& Rinaudo, C. (2012). Motivational and emotional profiles in university undergraduates: A self-determination theory perspective. The Spanish Journal of Psychology, 15(3), 1069-1080. https://doi.or g/10.5209/rev_SJOP.2012.v15.n3.39397

Grouzet, F. M. E., Kasser, T., Ahuvia, A., Dols, J. M. F., Kim, Y., Lau, S., ... Sheldon, K. M. (2005). The structure of goals across 15 cultures. Journal of Personality and Social Psychology, 89(5), 800-816. https://doi.org/ 10.1037/0022-3514.89.5.800

Kim, H., \& Choi, Y. (2015). Effects of creativity instruction activities on academic motivation and career maturity of university students: Based on NFTMTRIZ Creativity Education Model. Journal of Digital Convergence, 13(9), 277-286. http s://doi.org/10.14400/JDC.2015.13.9.277

Mancini, T., Caricati, L., Panari, C., \& Tonarelli, A. (2015). Personal and social aspects of professional identity. An extension of Marcia's identity status model applied to a sample of university students. Journal of Vocational Behavior, 89, 140-150. https://do i.org/10.1016/j.jvb.2015.06.002

Migone de Faletty, R., \& Moreno, E. J. (1999). Cuestionario de Motivaciones Ocupacionales (CUMO) (1.a ed.). Buenos Aires: Universidad del Salvador, Instituto de Investigaciones Psicoantropológicas.
Navarro Guzmán, C., \& Casero Martínez, A. (2012). Análisis de las diferencias de género en la elección de estudios universitarios. Estudios Sobre Educación, 22, 115-133. Recuperado de https://www.unav.edu/publi caciones/revistas/index.php/estudios-sobre -educacion/article/view/2075

Rovella, A., Sans de Uhrlandt, M., Solares, E., Delfino, D., \& Diaz, D. (2008). Motivo de logro y elección de la carrera de psicología. Revista Electrónica de Psicología Política, 6(18), 93-100. Recuperado de http://www.psicopol.unsl.e du.ar/Diciembre2008 Nota5.pdf

Ryan, R., \& Deci, E. (2000). Intrinsic and extrinsic motivations: Classic definitions and new directions. Contemporary Educational Psychology, 25(1), 54-67. https: //doi.org/10.1006/ceps.1999.1020

Skatova, A., \& Ferguson, E. (2014). Why do different people choose different university degrees? Motivation and the choice of degree. Frontiers in Psychology, 5, 1-15. http s://doi.org/10.3389/fpsyg.2014.01244

Troiano, H., \& Elias, M. (2014). University access and after: Explaining the social composition of degree programmes and the contrasting expectations of students. Higher Education, 67(5), 637-654. https:// doi.org/10.1007/s10734-013-9670-4

Valle Arias, A., Rodríguez, S., González Cabanach, R., Núñez Pérez, J. C., González Pienda, J. A., \& Rosario, P. (2010). Perfiles motivacionales y diferencias en variables afectivas, motivacionales y de logro. Universitas Psychologica, 9(1), 109-122. Recuperado de http://revistas.javeriana.ed u.co/index.php/revPsycho/article/view/283

Ye, L. H. (2015). Work values and career adaptability of Chinese university students. Social Behavior and Personality, 43(3), 411-422. https://doi.org/10.2224/sbp.2015. 43.3.411

\section{Notas}

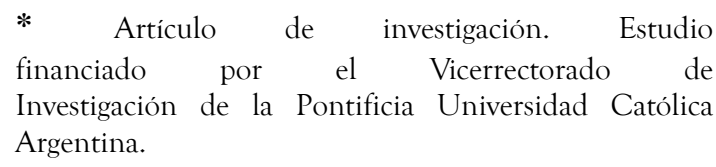

